\title{
Japan plans revision of science lessons
}

David Cyranoski, Tokyo

Alarmed by declining student interest in science and the superior test scores of some of its neighbours in east Asia, Japan is planning to reform the way science is taught in its schools.

"In the past we focused on making sure that everyone was getting a standard education," says a government official involved in the reforms. "Now we want to focus on enhancing the respective talents of each student."

The plan will start next year with the establishment of 20 'super-science high schools', each of which will receive special funding to buy equipment and hire teaching assistance from university researchers. The schools will also form 'science clubs' and confer with the universities on teaching methods and curriculum content. In addition, the plan will provide small grants for equipment to 1,500 other schools.

Yozo Shimomura, an Earth sciences teacher at Shinagawa Etoile Girls' High School in Tokyo, says he hopes that the plan will help to encourage independent thought among students. But critics have expressed concern that overworked teachers in Japan's

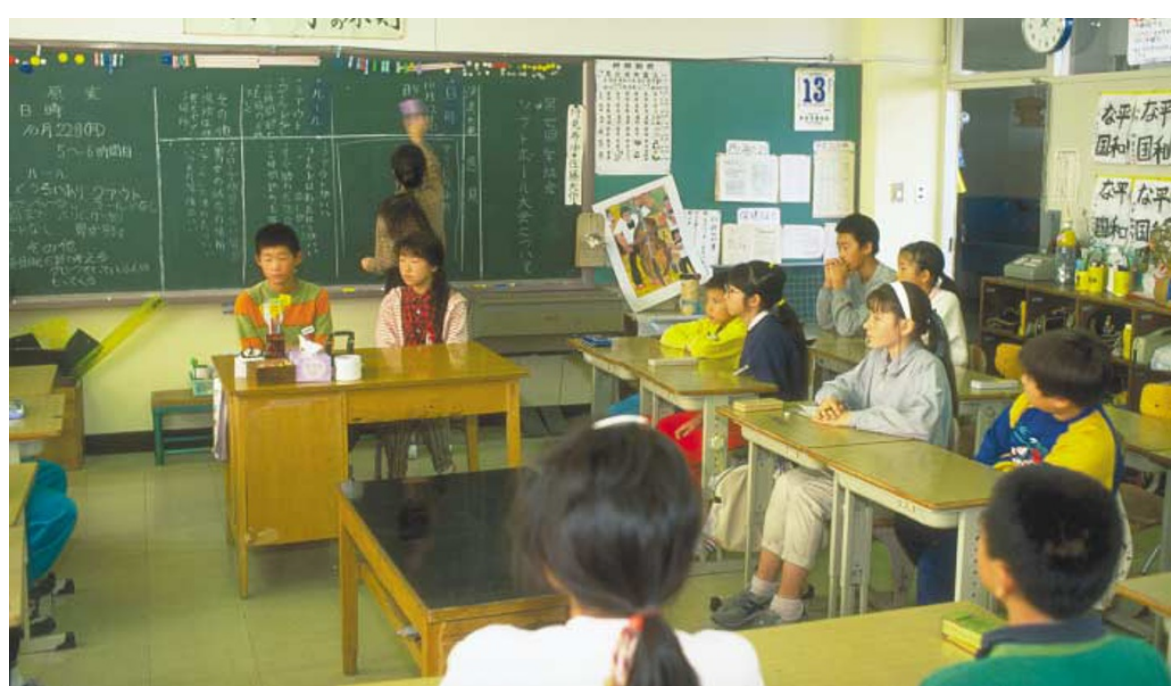

Learning shortfall: children's test scores in Singapore, Taiwan and Korea are surpassing those in Japan.

large classrooms need far more help than the package of measures is likely to offer.

Standardized tests of 13-14-year-olds indicate that children in Singapore, Taiwan and Korea are doing better than those in Japan. And surveys conducted at the same time as the tests found that the number of
Japanese students who enjoy science or would like to make a career out of it is among the lowest in the countries tested.

Japan's newly formed Ministry of Education, Culture, Sports, Science and Technology is seeking $¥ 9$ billion (US $\$ 75$ million) in its budget request for the reforms.

\section{Ministry attempts to breathe life into clinical trials}

\section{David Cyranoski, Tokyo}

The Japanese government has unveiled fresh incentives that it hopes will encourage doctors and researchers to participate in clinical trials of combination therapies.

According to statistics from the Ministry of Health, Labour and Welfare, the number

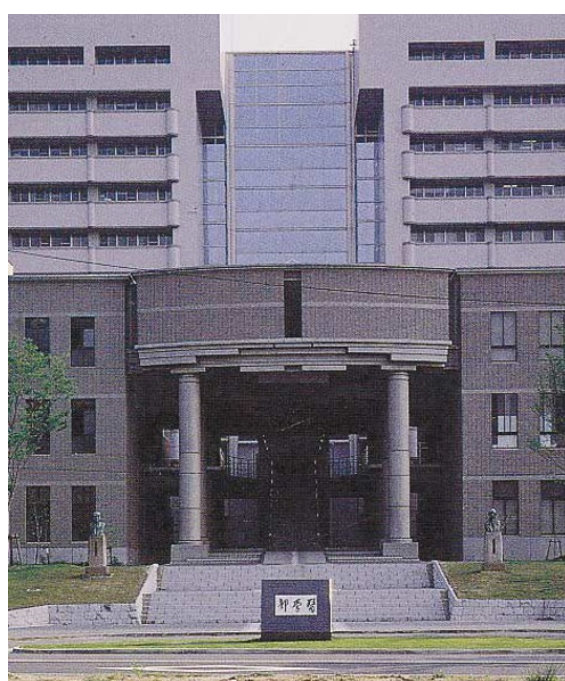

The scheme could encourage trials at institutes such as Osaka University's medical school. of trials sponsored by the drugs industry in Japan has fallen by half over the past five years. The ministry is to introduce a grant scheme that will provide $¥ 10$ billion (US\$84 million) next year for clinical trials.

Toshiro Nakagaki, an official in the health ministry, says that the new scheme will help to reverse this decline and will build up Japan's capacity for conducting drug trials. The grants, which will probably last for three years, will be available to teams of doctors working on various diseases, including cancer, heart disease and diabetes.

The fall in the number of clinical trials is blamed on several botched investigations. For example, in 1993, three patients died during a trial for the shingles treatment sorivudine - and a further 15 died after the drug was approved - because it reacted with their fluorouracil anticancer treatment.

Tough new procedures for informed consent, implemented in 1998, have also hampered the organization of clinical trials. Doctors who take part have to work through the necessary paperwork during their already-crowded working hours, says Shigenobu Kanba, a neuropsychiatrist at Yamanashi Medical University.

And the trials can take up to five or six years to complete, according to Kanba. As a result, many drug developers turn to contract research organizations that can do tests more quickly and cheaply abroad. "Some companies do all their research overseas," says Keiko Oishi, head of international relations at CMIC, a Tokyo-based company that contracts out clinical trials on behalf of pharmaceutical companies.

The past couple of years have shown some signs of improvement. New patientrecruitment centres and the recent legalization of recruitment advertisements have produced more willing patients for trials. "Increasingly, companies are realizing that for practical reasons, it is necessary and possible to do trials in Japan," says Oishi.

But the key problem is doctors' reluctance to participate. "Until now, doctors have thought of clinical trials as a lowly part-time job, and papers or successful trials don't add to the list of a doctor's achievements," says Kanba.

The grants to be made available under the new ministry programme could help, says Oishi. They can be used, for example, to pay for clinical-research coordinators who will help to recruit patients and take care of informed-consent procedures. They will also finance any extra tests the doctors might have to perform during the trials. 